\title{
Spatial and temporal characterization of sea-ice deformation
}

\author{
Jennifer K. HUTCHINGS, ${ }^{1}$ Andrew ROBERTS, ${ }^{1}$ Cathleen A. GEIGER, ${ }^{2}$ \\ Jacqueline RICHTER-MENGE ${ }^{3}$ \\ ${ }^{1}$ International Arctic Research Center, University of Alaska Fairbanks, 930 Koyukuk Drive, Fairbanks, AK 99775-7340, USA \\ E-mail: jenny@iarc.uaf.edu \\ ${ }^{2}$ Department of Geography, University of Delaware, Newark, DE 19716, USA \\ ${ }^{3}$ US Army Cold Regions Research and Engineering Laboratory, 72 Lyme Road, Hanover, NH 03755-1290, USA
}

\begin{abstract}
In late March 2007 an array of GPS ice drifters was deployed in the Beaufort Sea as part of the Sea Ice Experiment: Dynamic Nature of the Arctic (SEDNA). The drifters were deployed in an array designed to resolve four, nested spatial scales of sea-ice deformation, from 10 to $140 \mathrm{~km}$, with the arrays maintaining appropriate shape for strain-rate calculation until mid-June. In this paper, we test whether sea-ice deformation displays fractal properties in the vicinity of SEDNA. We identify that deformation time series have different spectral properties depending on the spatial scale. At the scales around $100 \mathrm{~km}$, deformation is a red-noise process, indicating the importance of the ice-pack surface forcing in determining the deformation rate of sea ice at this scale. At smaller scales, the deformation becomes an increasingly whiter process (it has pink noise properties), which suggests an increasing role of dissipative processes at smaller scales. At spatial scales of $\mathbf{1 0 - 1 0 0 ~} \mathrm{km}$, and sub-daily scales, there is no deformation coherence across scales; coherence only becomes apparent at longer scales greater than $100 \mathrm{~km}$. The lack of coherence at small scales aids in understanding previous observations where correlation between $10 \mathrm{~km}$ regions adjacent to each other varied widely, with correlation coefficients between -0.3 and 1 . This suggests it is not appropriate to think of sea ice as having a decorrelation length scale for deformation. We find that lead scale observations of deformation are required when estimating ice growth in leads and ridging time series. For the two SEDNA arrays, we find coherence between 140 and $20 \mathrm{~km}$ scale deformation up to periods of $\mathbf{1 6}$ days. This suggests sea-ice deformation displays coherent deformation between $100 \mathrm{~km}$ scale and the scale of the Beaufort Sea (of order $1000 \mathrm{~km}$ ), over synoptic time periods (daily to weekly timescales). Organization of leads at synoptic and larger scales is an emergent feature of the deformation field that is caused by the smooth variation of surface forcing (wind) on the ice pack.
\end{abstract}

\section{INTRODUCTION}

During winter, sea-ice deformation, including horizontal opening, closing and shearing, occurs at leads and ridges. These are linear features, tens of kilometers long, which can organize into lead systems (linear kinematic features (LKFs)) that run hundreds of kilometers across the Arctic (Schulson and Hibler, 1991; Kwok, 2003). The ice pack is highly fractured. In synthetic aperture radar analysis (Kwok, 2003) it is observed to undergo continuous deformation at $10 \mathrm{~km}$ scales, and the most energetic deformation is confined to narrow LKFs, and is an order of magnitude larger than the deformation outside LKFs. The spacing between leads varies depending on the confining stress on the ice pack and icepack strength (Hutchings and others, 2005), and also displays multiple scales of organization. Overland and others (1995) demonstrate that the ice pack in the Beaufort Sea has $\sim 10 \mathrm{~km}$ spacing between leads, and LKF spacing can be considered to be an order of magnitude larger. It can be argued that this behaviour either demonstrates an inherent scaling law for sea-ice deformation (such as discussed by Schulson and Hibler, 1991; Weiss and Marsan, 2004), or demonstrates differing mechanisms controlling ice dynamics at large and small scales (Overland and others, 1995; McNutt and Overland, 2003).

Analysis of strain-rate products from RADARSAT analyses and ice-drifting buoys by the team of Marsan, Weiss, Rampal, Stern and Lindsay (Marsan and others, 2004; Rampal and others, 2008; Stern and Lindsay, 2009) demonstrates that sea-ice deformation is a fractal process in space and time. Marsan and others (2004) identify that sea-ice deformation is localized, as deformation is concentrated at linear features. These results follow observations that leads and floe sizes follow a fractal distribution, and suggest that the mechanism controlling sea-ice failure is scale-invariant. The results, however, do not demonstrate why Overland and others (1995) identified a hierarchy in the organization of leads in the Beaufort Sea. Determining the correct distribution for sea-ice deformation (active leads and ridges) is critical for quantifying surface heat fluxes and new ice formation (Geiger and Drinkwater, 2005).

In this paper, we reproduce the Marsan and others (2004) and Rampal and others (2008) results with an array of icedrifting buoys that was designed to measure sea-ice deformation over cascading scales and relate the observed scaling relationships to changing weather. This unique, nested buoy array operated from late winter until early summer 2007. We investigate how deformation of the ice pack within the buoy array varies over length scales from 10 to $140 \mathrm{~km}$ and timescales of hours to days. The impact of ice-drift sampling resolution, in space and time, on estimates of first-year ice growth is investigated. This applies the results of Marsan, Weiss, Rampal, Stern and Lindsay to test a deformation monitoring capturing sea-ice properties such as ice growth and open-water fraction. Recommendations for the design of sea-ice deformation monitoring systems are provided. We investigate if pack-ice deformation has a decorrelation length scale, and show how changing weather patterns impact the distances over which coherent deformation is experienced. 


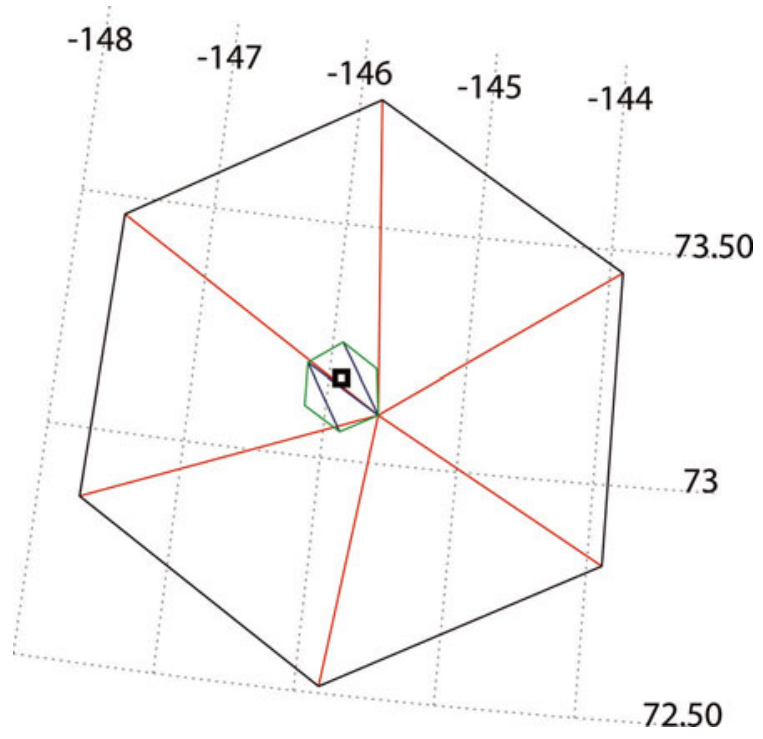

Fig. 1. Map of GPS buoy positions on 25 March at 00:00Z. Buoy arrays are outlined in colour, referring to the spatial scale set to which the array belongs. The largest array, $140 \mathrm{~km}$ scale, is outlined in black. Six $70 \mathrm{~km}$ scale arrays are red. There is one green $20 \mathrm{~km}$ scale array and four blue $10 \mathrm{~km}$ scale arrays. The camp location is marked with a black square.

Wavelet cross-coherence analysis of divergence on two spatial scales within the same region provides insight into emergent patterns in ice deformation. This sheds light on why leads appear to be organized on $>100 \mathrm{~km}$ scales. Our findings are summarized with suggestions on how to improve modelling and observation of sea-ice deformation.

\section{FIELD CAMPAIGN}

The Sea Ice Experiment: Dynamic Nature of the Arctic (SEDNA) field campaign utilized the Applied Physics Laboratory Ice Station (APLIS) in the Beaufort Sea in spring 2007. Two arrays of GPS ice drifters were deployed on 24 March in nested hexagons, of width 20 and $140 \mathrm{~km}$, around APLIS (Fig. 1). All 12 buoys drifted with the ice pack and transmitted data until 22 June. These arrays formed the backbone of SEDNA (described in detail by Hutchings and others, 2008; Hutchings, 2009).

The GPS buoy positions are used to calculate strain-rate components, divergence and maximum shear rates (Fig. 2) of the ice pack within the array. The method we use, and error analysis, is outlined by Hutchings and Hibler (2008). The SEDNA GPS buoys had a position error less than $10 \mathrm{~m}$. Propagation of error analysis throughout strain-rate calculations indicates that strain-rate error is an inverse function of area enclosed by the buoy array and ice velocity. Errors are largest for small arrays moving slowly, and the signal-tonoise ratio becomes unacceptably small for arrays smaller than $3 \mathrm{~km}^{2}$ moving at $<0.002 \mathrm{~m} \mathrm{~s}^{-1}$. We flag, and do not include in our analysis, estimated strain rates that do not meet these two requirements, and estimate our strain-rate error as $<1 \times 10^{-8} \mathrm{~s}^{-1}$. The two arrays provide time series of ice-pack divergence and shear over four spatial scales that vary between 10 and $140 \mathrm{~km}$. This is a unique dataset with which we can investigate the spatial scales of sea-ice deformation coherency. It is also useful for identifying if there is a scaling relationship linking the statistical properties
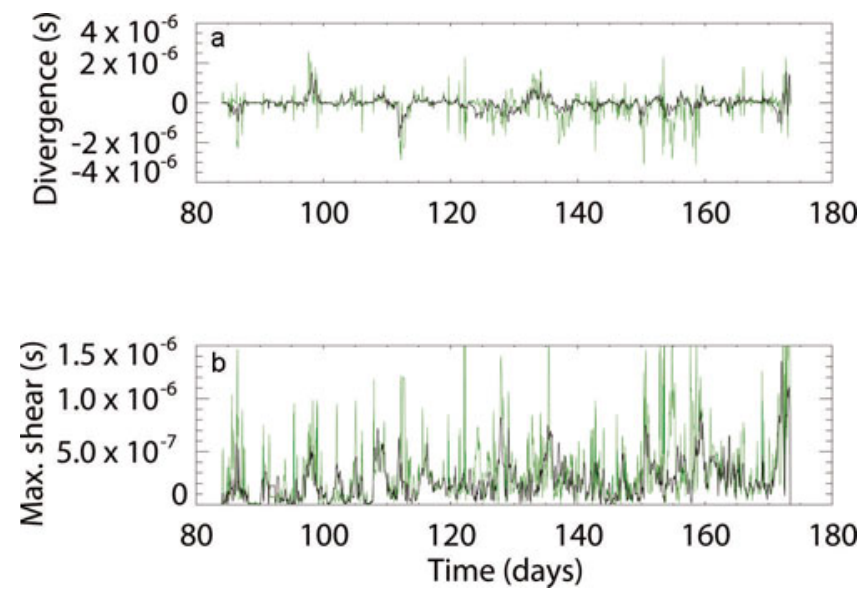

Fig. 2. Time series of (a) divergence rate, $\varepsilon_{l}$, and (b) maximum shear rate, $\varepsilon_{I I}$, for the $140 \mathrm{~km}$ scale array (black) and $20 \mathrm{~km}$ scale array (green). Total deformation rate is calculated as $\sqrt{\left(\varepsilon_{l}^{2}+\varepsilon_{\| l}^{2}\right)}$.

of sea-ice deformation and the scale over which the deformation is observed.

As buoys provide time series of sea-ice deformation we can investigate the temporal evolution of the coherence of the ice-pack deformation, over different scales, during the spring transition from a relatively strong, connected, concentrated ice pack to a disconnected summer pack. As the data were collected in late winter and spring, we cannot resolve the full seasonal variability in scaling properties of sea-ice deformation, which has been identified by Rampal and others (2008) and Stern and Lindsay (2009). However, our analysis does provide independent verification of the deformation scaling properties of a compact ice pack, previously identified by Marsan and others (2004).

The SEDNA deformation dataset has some features that are perhaps unusual and should be taken into consideration in following our analysis. At the start of the SEDNA campaign, a lead system opened in the $10 \mathrm{~km}$ region around the ice camp. These leads were visually observed to be up to $1 \mathrm{~km}$ wide at times, and froze into areas of thin level ice $(<50 \mathrm{~cm}$ thick by mid-April) that subsequently ridged. We estimate that all level ice that grew in the inner buoy array during the SEDNA experiment was ridged by mid-June. This is not the case in the larger array, which sampled several lead systems including the system that ran through the inner array. This event was unusual in that we were able to sample the impact of a single, relatively large, deformation event on the ice pack over a range of spatial scales using drifting buoys.

\section{SPATIAL SCALING OF STRAIN RATE}

SEDNA included nested buoy arrays (Fig. 1) that resolve icepack strain rate over a set of four spatial scales. For each buoy array we estimate strain-rate components and total deformation rate as described by Hutchings and Hibler (2008). The time series of total deformation are plotted as a function of the square root of array area (length) in Figure 3. Data are clustered into sets of different length scales: around $10 \mathrm{~km}$ (blue), $20 \mathrm{~km}$ (green), $70 \mathrm{~km}$ (red) and $140 \mathrm{~km}$ (black). For each length scale set, we calculated the statistical moments of the distribution of total deformation. In Figure 3 a least-squares fit to the means of each length scale set is shown. Mean deformation rate, $D$, scales logarithmically 


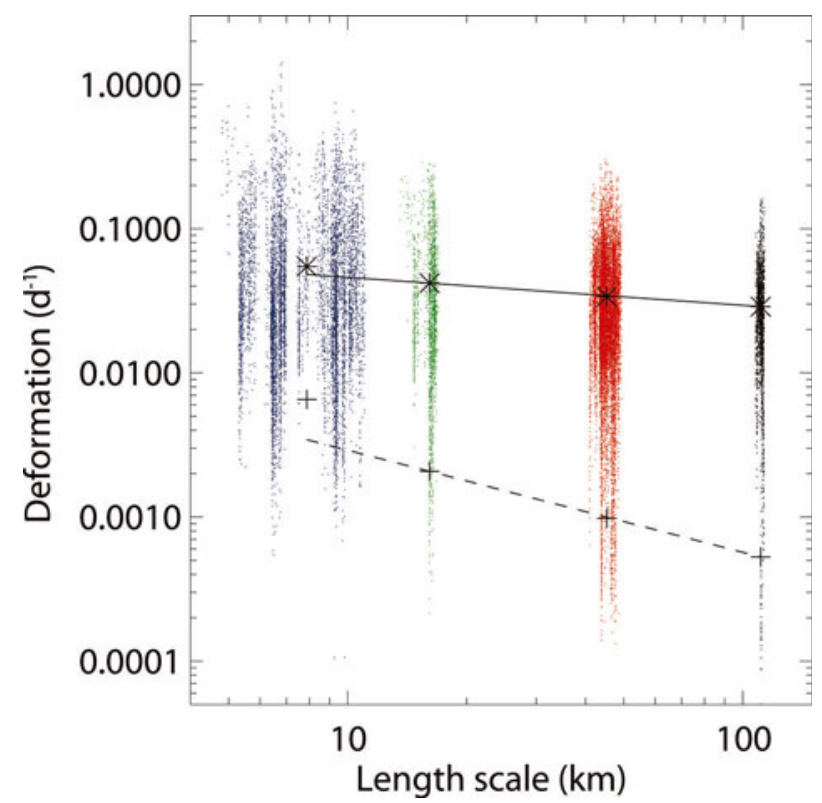

Fig. 3. All realizations of deformation rate and length scale (square root of area), for each sub-array in all sets, are plotted in the colours defined in Figure 1. Mean sub-array length scale and mean deformation is plotted (black stars) for each buoy sub-array set defined in Figure 1. The least-squares fit to these values is shown as a solid line. The variance of deformation for each sub-array is plotted (black crosses), and the dashed line is least-squares fit to these points.

with length scale, $L$, such that

$$
D \propto L^{H},
$$

where $H=-0.19$. Marsan and others (2004) found a similar relationship with an exponent of $H=-0.2$, for ice that covered the western Arctic during winter. Our result is an independent verification of the Marsan and others (2004) results. It can be argued these results show that the fractal nature of sea-ice deformation is experienced by a region of

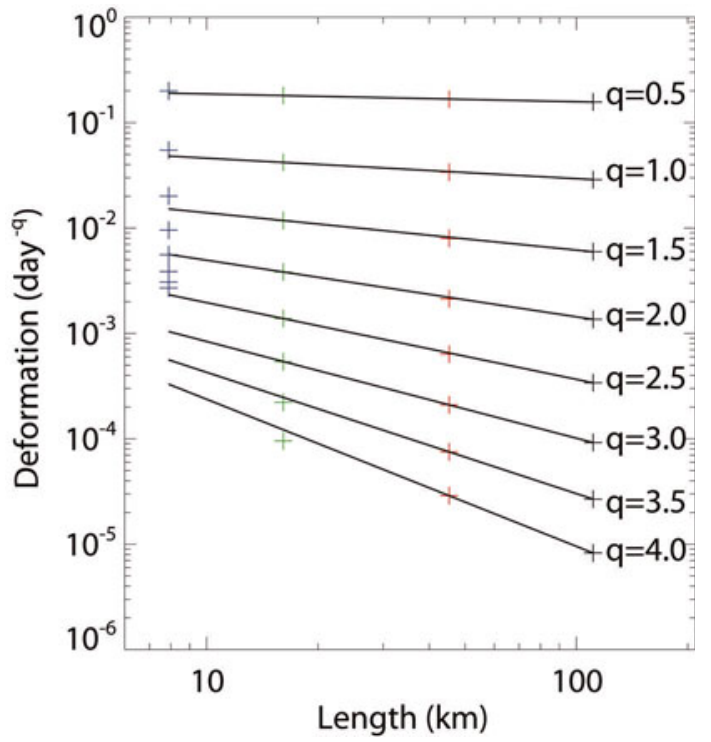

Fig. 4. Moments, $q$, between 0.5 and 4 , of deformation rate, $\left\langle D^{q}\right\rangle$, plotted against length scale. The colour of the mean value, plotted as crosses, corresponds to buoy array shown in Figure 1.

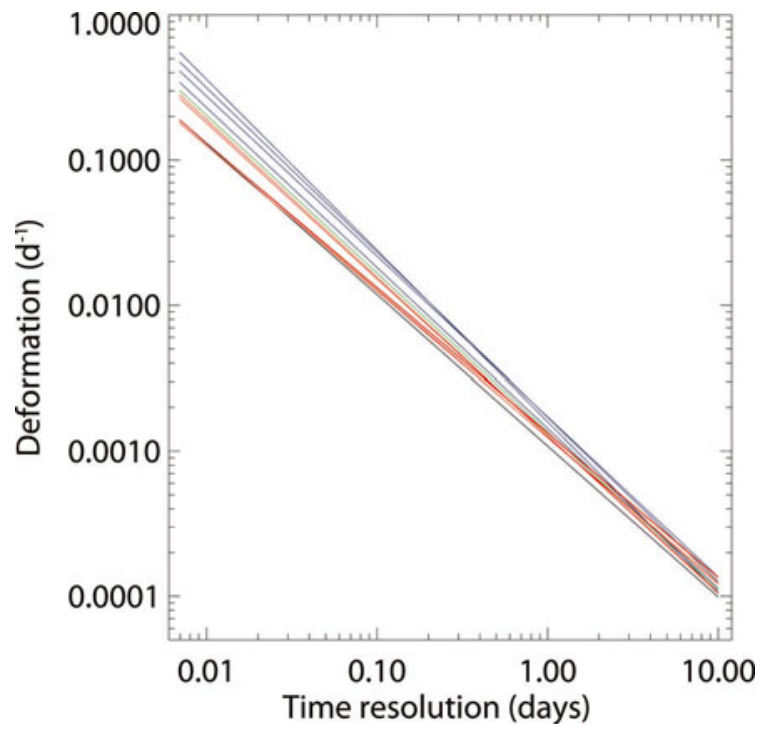

Fig. 5. Least-square fit to the means of deformation rate at each timescale sampled for all SEDNA sub-arrays. The colour corresponds to the spatial scale family the sub-array belongs to: $10 \mathrm{~km}$ (blue), $20 \mathrm{~km}$ (green), $70 \mathrm{~km}$ (red) and $140 \mathrm{~km}$ (black). The gradients of the smallest arrays are close to -1 .

ice as it evolves in time, as well as having a similar fractal distribution in space (Marsan and others, 2004). We also find that other statistical moments of deformation (standard deviation, skewness and kurtosis) show scaling properties, increasing as the spatial scale decreases. Like Marsan and others (2004), we find that sea-ice deformation displays multi-fractal properties (Fig. 4).

Our analysis supports the hypothesis of Marsan and others (2004) that sea-ice strain rate is localized. Like Marsan and others (2004), we find that as spatial scale decreases, larger strain rates become apparent, indicating the largest strain rates are accommodated by a small portion of the buoy array area. Another signature of this localization is that the variance of sea-ice deformation increases as spatial scale reduces, which we find in agreement with Marsan and others (2004). Stern and Lindsay (2009) found that this localization is a property of the spatial structure of the deformation field.

\section{TEMPORAL SCALING}

For each buoy position time series, we can subsample the time series, to calculate deformation time series with varying temporal resolution. The highest resolution available is $10 \mathrm{~min}$. The lowest resolution we subsample at is 10 days. This provides three orders of magnitude in temporal resolution for which we can search for the existence of a temporal scaling relationship. Similar scaling relationships (Fig. 5) are found for all sub-arrays, which have spatial scales varying from 6 to $140 \mathrm{~km}$.

The first observation we can make from Figure 5 is that there is a log-log linear scaling relationship between deformation and timescale, $T$, where $D \propto 1 / T$, such that the evolution of deformation in time can be thought of as a pink noise process. Sea-ice deformation at spatial scales between 6 and $140 \mathrm{~km}$ displays non-equilibrium critical behaviour. This is an important observation for those wishing to model sea-ice deformation. 
At the highest and lowest resolutions the scaling behaviour falls away from linear. This is more apparent in section 4.1, but we comment on this here as it does affect our interpretation of the data. At high temporal resolutions, from $10 \mathrm{~min}$ to $<1$ hour, the GPS position error propagated through our calculations becomes similar in magnitude to the size of the deformation, so at these higher resolutions the scaling relationship represents GPS noise. There is a lower timescale limit over which deformation can be reliably calculated with GPS, and this limit varies from $30 \mathrm{~min}$ to 1 hour across the spatial scales resolved in SEDNA. Smaller spatial scales have a larger lower temporal sampling limit.

For the lower temporal resolutions, above daily sampling, we find the scatter in mean deformation displays large variability. The gradient of the scaling relationship appears to increase, becoming more of a red-noise process, and for some sub-arrays the magnitude of deformation increases. This excursion from the scaling relationship experienced at timescales between 1 hour and 1 day appears small $(<10 \%$ of mean deformation rate). However, in section 4.1 it is demonstrated that these small deviations have a dramatic impact on estimation of ice growth in leads.

Spectra of total deformation rate, for sub-arrays of varying spatial scale, are plotted in Figure 6. These spectra indicate, like Figure 5, that deformation is a pink noise process. Unlike Figure 5, Figure 6 encompasses all information about the deformation statistical distribution (Figure 5 only provides information about the statistical mean). Comparison of Figures 5 and 6 suggests that the quantity investigated in Figure 5, mean deformation, does not represent the full scaling behaviour of sea-ice deformation time series (sea-ice deformation has a non-Gaussian distribution, with statistical moments that vary with space- and timescales). This suggests that spectral analysis techniques may be more appropriate for investigating the scaling properties of sea-ice deformation than simply considering the scaling properties of the mean.

The degree of whitening of the spectra increases as length scale reduces. There is no apparent universal scaling parameter for sea-ice deformation that applies across spatial scales from 10 to $70 \mathrm{~km}$. To understand this, we need to consider the following point: if ice rheology is not significant in the force balance on the ice pack, we expect sea-ice velocity and deformation time series to follow atmospheric and oceanic forcing on the ice pack. Hence we would expect the time series of deformation to possess red spectral character. Ice interaction acts to dissipate energy, whitening the spectra of deformation. This suggests we are seeing the greatest energy dissipation at the smallest length scales, which resolve only one or two leads. As length increases, more lead systems are encompassed in the buoy array. We know that these lead systems develop in patterns that are, to first order, determined by the wind forcing on the entire Beaufort Sea ice pack (Walter and Overland, 1993) or at least to the $1000 \mathrm{~km}$ (synoptic) scale of atmospheric synoptic systems (Geiger and others, 2000); and that these lead patterns have fractal characteristics (Weiss and Marsan, 2004). It follows that if we sample the ice-pack deformation below the synoptic spatial scale, ice deformation might show self-organized critical behaviour (as shown in Figs 5 and 6) and that organization increases as the length scale increases and lead patterns, associated with particular storms, become apparent. Figure 6 indicates that organization of the sea-ice deformation field reflects atmospheric variability over length scales of $140 \mathrm{~km}$. At smaller resolutions, there is an

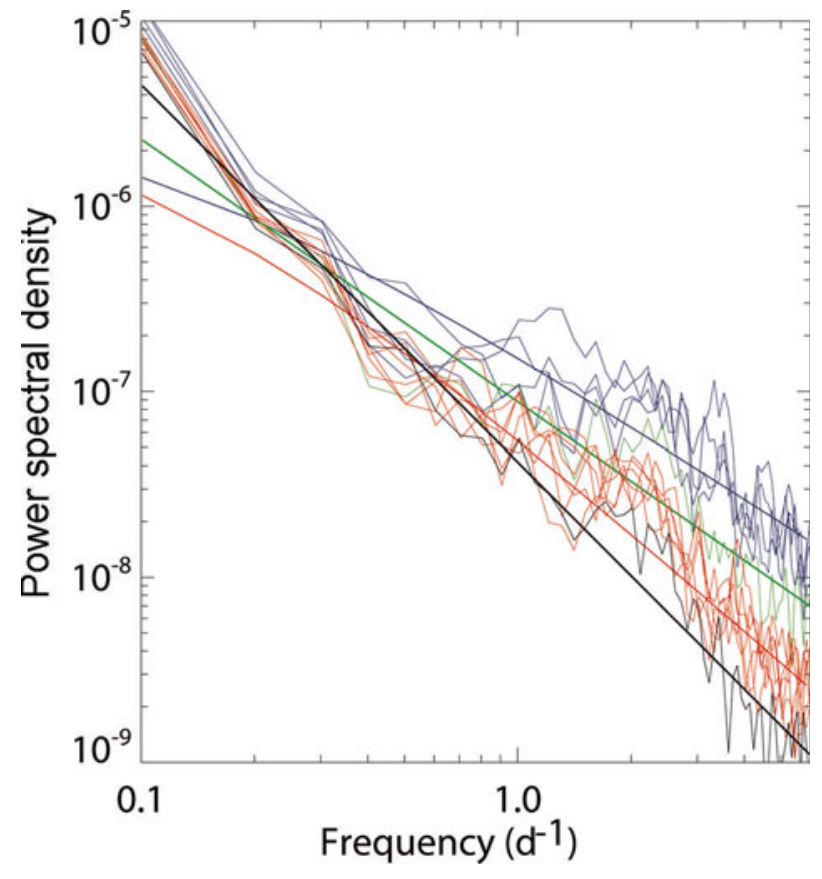

Fig. 6. Spectral density of deformation, estimated with a fast Fourier transform method (Jenkins and Watts, 1969). Spectra are calculated from each sub-array, and mean log-log linear fit to spectra with spatial scales of $10 \mathrm{~km}$ (blue), $20 \mathrm{~km}$ (green), $70 \mathrm{~km}$ (red) and $140 \mathrm{~km}$ (black) are plotted. At the largest spatial scale, $140 \mathrm{~km}$, the spectra can be approximated by red noise. The other spectra are pink, becoming whiter as spatial scale decreases. At the largest spatial scale, $140 \mathrm{~km}$, the spectra have a slope of -2 , and this slope decreases with reducing spatial scale: $-1.8(70 \mathrm{~km}),-1.4(20 \mathrm{~km})$ and $-1.3(10 \mathrm{~km})$.

increasing randomness in the deformation field sampled, and our results suggest that individual lead deformation may be close to white noise.

\subsection{Impact of spatio-temporal resolution on mass- balance estimates}

The buoy array area or divergence time series can be used to estimate new ice growth in leads and redistribution of this new ice to ridges. To do this we use a model of thermodynamic ice growth and redistribution that is driven by the observed buoy array divergence. This model uses climatological ice-growth/-melt rates from Maykut and Untersteiner (1971) and the assumption that thinnest ice is ridged preferentially. Figure 7 shows a time series of new level ice and ridged ice volume estimated for the large $140 \mathrm{~km}$ scale array, with hourly temporal resolution.

The localization and fractal character of ice-pack strain rate is a result of the ice-pack deformation occurring at leads and ridges. Divergence of arctic sea ice increases in mean magnitude and variance as the spatial scale of estimated divergence reduces. This means that measurements taken over smaller scales will result in larger estimates of lead opening and closing rates. It affects estimation of new ice growth. In Figure 8, we plot the area-averaged mean thickness of ice grown between 26 March and 22 June as a function of array length scale.

Figure 8 indicates that our calculation of new ice growth has fractal properties, like lead area and divergence. The smaller the length scale over which divergence and growth is estimated, the larger the estimated new ice thickness. 

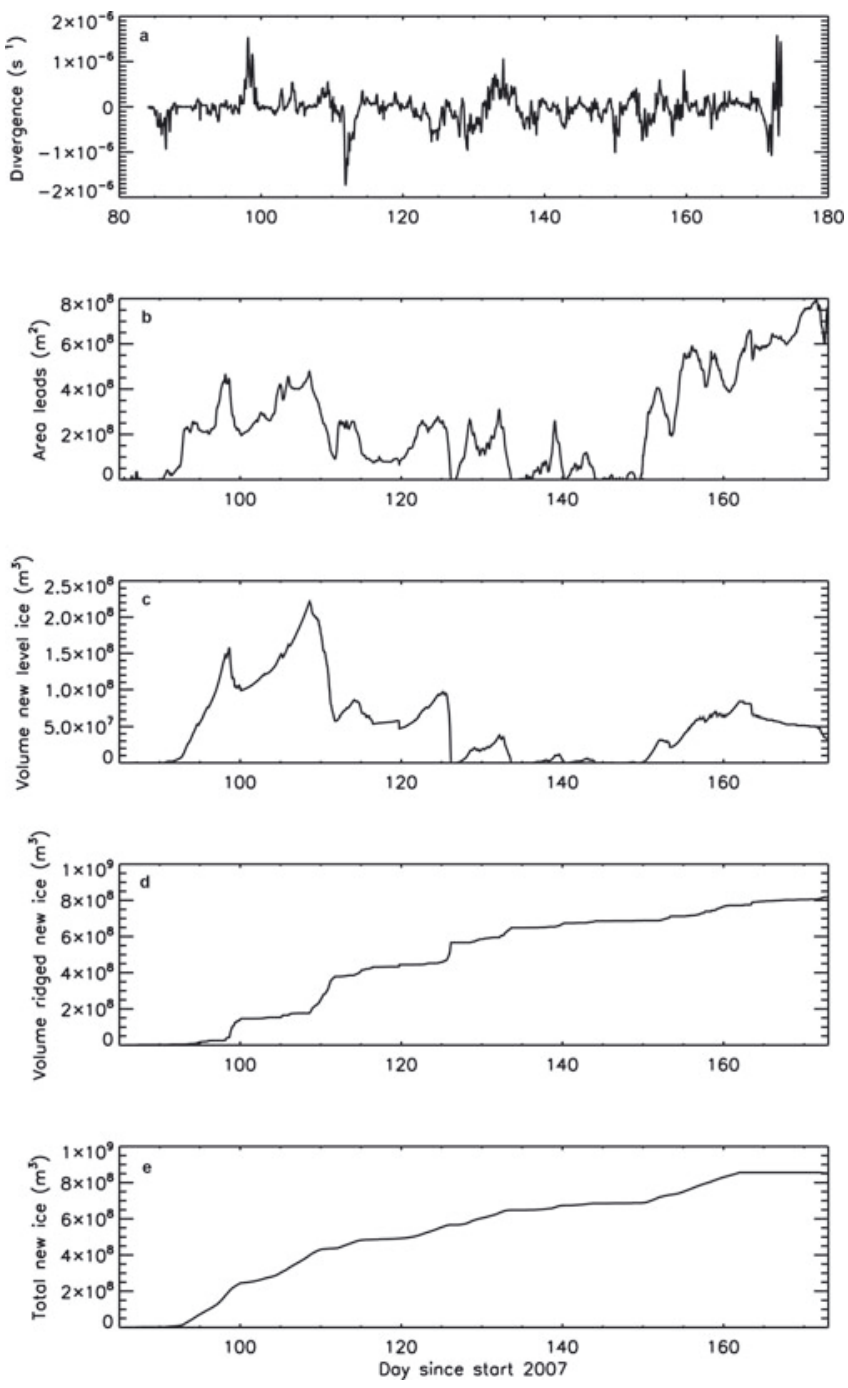

Fig. 7. Divergence of the largest SEDNA array, between 26 March and 22 June (a), is used to calculate open-water fraction (b) and ridged ice area. A model of ice growth, using Maykut and Untersteiner (1971) growth rates, tracks the thickness of ice grown in leads, in $200.5 \mathrm{~m}$ thickness categories. On closing, thin ice is ridge preferentially, and subsequently tracked as ridged ice thickness. Time series of modelled level ice volume (c) and ridged ice volume (d) are shown, and the sum of these (the total volume of new ice) is shown (e).

Clearly, large-scale deformation measurements underestimate the open-water fraction, closing rate and hence new ice growth. To prevent this problem, either individual leads must be resolved in the measured deformation field (requiring observations at better than $10 \mathrm{~km}$ spatial scales), or some distribution of lead divergence, perhaps a fractal distribution, must be assumed over the region.

Temporal resolution also has an impact on ice growth calculations. In Figure 9 we show the relationship between new ice growth and time resolution for all SEDNA subarrays. Over the timescales which we have identified to be not affected by GPS noise and low-resolution sampling issues ( 1 hour to 1 day), we see a scaling relationship that is similar for all spatial scales.

Sub-hourly resolutions introduce increasing errors into the deformation calculation, through GPS noise, so ice growth is not correctly estimated. Note that other measurement methods for ice position and deformation have larger

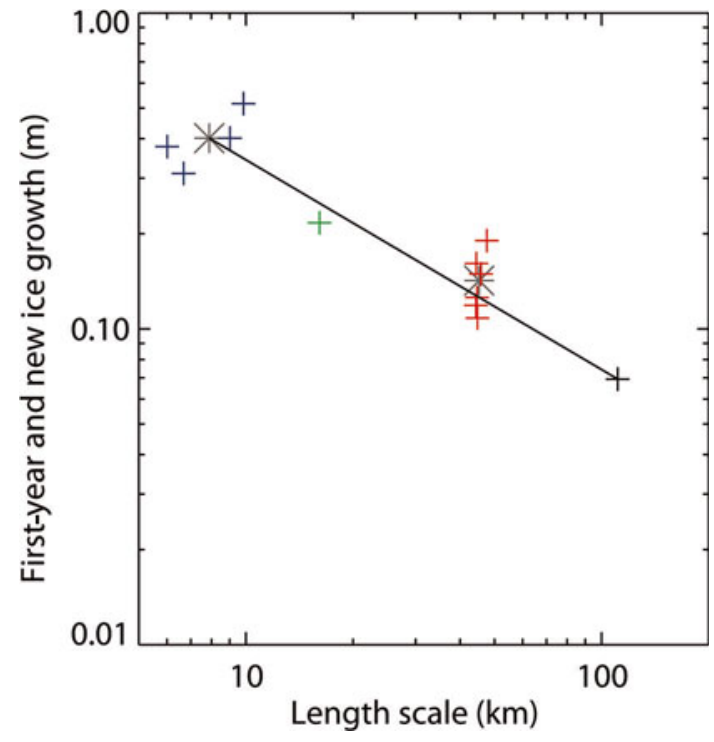

Fig. 8. Area-averaged mean thickness of ice grown between 26 March and 22 June, as a function of array length scale (the square root of mean array area). Mean values for the 10 and $70 \mathrm{~km}$ arrays are plotted as stars. The least-squares fit has a gradient of $-0.7(\mathrm{~m}) / \log (\mathrm{km})$.

errors than GPS (Advanced Research and Global Observation Satellite (ARGOS) position error is $\sim 300 \mathrm{~m}$ and the RADARSAT Geophysical Processing System position error is $320 \mathrm{~m}$ (Lindsay and Stern, 2003)), so they cannot resolve sea-ice deformation below 3 hours (Hutchings and others, unpublished information). The calculation of new ice growth in leads, and scales that can be resolved, is impacted by the divergence measurement error such that measurements with lower accuracy have a large temporal sampling cut-off and will tend to overestimate ice growth.

At lower resolutions the changing ice growth rate due to thickening in the Maykut and Untersteiner (1971) model is insufficiently resolved, resulting in overestimation of ice thickness in an opening pack. Between hourly and daily resolutions, where our calculations are reliable, we see smaller ice growth rates at higher resolutions because the greater fluctuation in ice-pack divergence resolved at these scales retards ice growth. Figure 9 indicates the importance of resolving the temporal evolution of leads in sea-ice massbalance estimation.

Basal ice growth during our experiment period was $\sim 0.2 \mathrm{~m}$. New ice growth varied between half and five times the basal growth rate, depending on the spatial and temporal scale over which new ice growth was estimated. This new ice is a significant part of the winter ice growth in the Beaufort Sea. Correct representation of it in models and accurate observations of new ice growth are important for correctly representing the sea-ice mass balance.

\subsection{Are spatial and temporal scaling relations independent?}

A natural question following from our separate investigation of spatial and temporal sampling is: 'is the spatial scaling relationship robust to temporal sampling?'. We find that it is not, as did Rampal and others (2008) and Geiger and Drinkwater (2001). Figure 10 shows $H$, from Expression (1), estimated for deformation that has been calculated with different temporal resolutions. We find a trend where the 


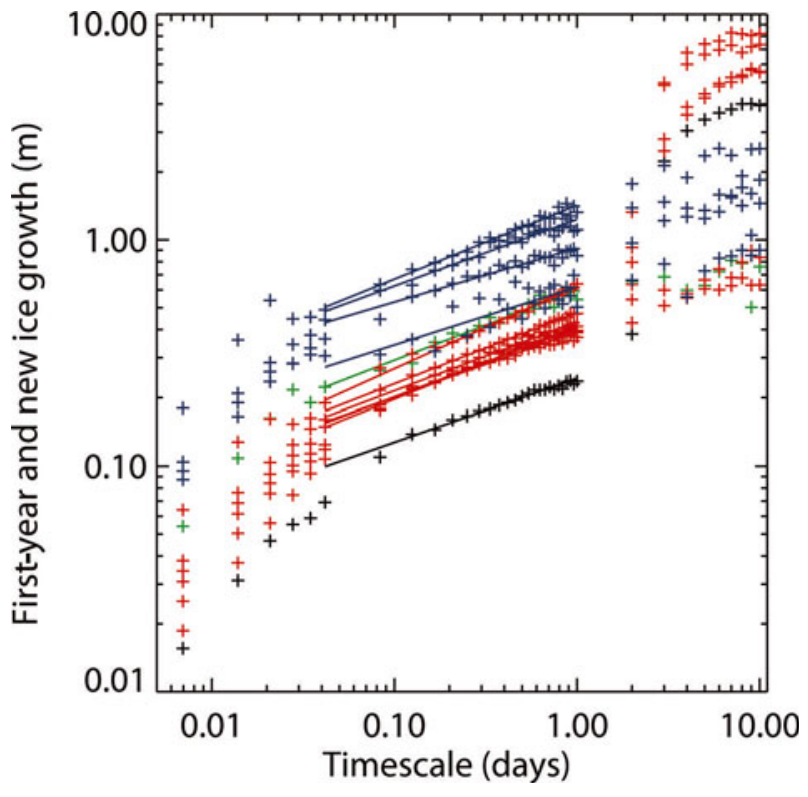

Fig. 9. Area-averaged mean thickness of ice grown between 26 March and 22 June $2007(G)$, as a function of timescale $(T)$ resolved. Mean values for all arrays are plotted as crosses. Each solid line is a least-square fit to the mean ice growth in each subarray. These lines follow the relationship $G \propto T^{\beta}, \beta=0.30$ with standard deviation 0.03 . The colours correspond to the spatial scale set to which the array belongs: $10 \mathrm{~km}$ (blue), $20 \mathrm{~km}$ (green), $70 \mathrm{~km}$ (red) and $140 \mathrm{~km}$ (black).

magnitude of $H$ decreases as timescale increases, as did Rampal and others (2008). This suggests a whitening of the spatial scaling character of sea-ice deformation as temporal resolution decreases, suggesting that greater random noise is introduced into the scaling estimate as the number of buoy positions used to calculate the deformation time series is reduced. Geiger and others (2000), Geiger and Drinkwater (2005) and Rampal and others (2008) point out similar coupling between spatial and temporal scaling relations for sea-ice deformation across the Arctic, in both summer and winter. The observation that there is a relationship between temporal and spatial scaling means that we can relate measurements of sea-ice deformation over different scales, as has been done by Geiger and others (2000) and Geiger and Drinkwater (2005) using a different approach to the one we take in section 4.3. It also means we can investigate the length scales over which ice-pack deformation is coherent given a time series of sea-ice deformation measured at a variety of spatial scales.

\subsection{Spatio-temporal scaling of coherence of divergence}

Ice velocity and strain rate do not behave as stationary processes. Their statistical properties, including mean and standard deviation, evolve over synoptic and seasonal timescales. We also know that divergence between buoy arrays that are closely spaced, of magnitude $10 \mathrm{~km}$, has correlation that exhibits large spatial variation, varying from weakly anticorrelated, to uncorrelated to strongly correlated over short distances, $<70 \mathrm{~km}$ (Hutchings and Roberts, in press). The degree to which the two hexagonal SEDNA buoy-array divergence time series are correlated varies by up to $50 \%$ depending on the time period over which the correlation analysis is performed. This suggests a classic

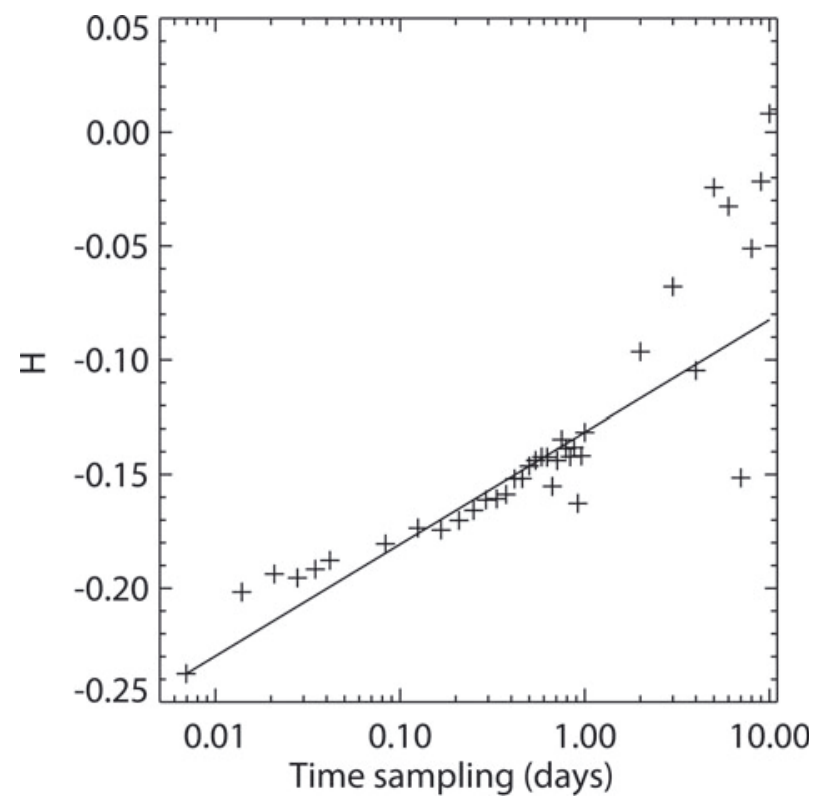

Fig. 10. Gradients calculated as in Figure 3, for time sampling that varies between $10 \mathrm{~min}$ and 10 days. A least-square fit to values of $H$ and $\log T$ is shown. The gradient of this fit is 0.5 (log day) and only varies by \pm 0.1 (log day) if the least-square fit is confined to less time-sampling spread.

decorrelation length scale is not appropriate for sea-ice deformation.

Figure 11 provides the wavelet cross-coherence between large $(140 \mathrm{~km})$ and small $(20 \mathrm{~km})$ scale divergence. This has been calculated following the methods of Grinsted and others (2004) using a sixth-order derivative of Gaussian wavelet (DOG6) with 0.25 octaves per scale. This analysis reveals that there is significant coherence over synoptic weather-scale periods. There are specific features in Figure 11 that are insightful for understanding the changing coherence length scale for sea-ice deformation, and we discuss the four main points here.

1. Significant power in both divergence and ice velocity is experienced for the semi-diurnal time period, driven by inertial motion of the ice and ocean. During late winter the magnitude of this semi-diurnal power is low (Hutchings and Roberts, unpublished information), but there are times when a semi-diurnal oscillation in divergence is present. During some of these times, there is coherence between divergence for small and large SEDNA arrays. Our main finding is that the times over which divergence is responding with a semi-diurnal period are sporadic and last for $<2$ days. This suggests that, in the Beaufort Sea in winter, inertial motion has a secondary impact on sea-ice deformation, and winds are more important in the force balance on the ice pack.

2. Coherence in ice divergence is experienced across the $140 \mathrm{~km}$ SEDNA region over synoptic time periods (3-8 days), for the majority of the time series. There are, however, three times when divergence becomes incoherent: 30 April-2 May, 5-10 May and 10-15 June.

3. During the first part of the time series, divergence is coherent across the $140 \mathrm{~km}$ SEDNA region for time periods longer than the synoptic timescale. This suggests a degree of connectivity of the ice pack, whereby ice is 


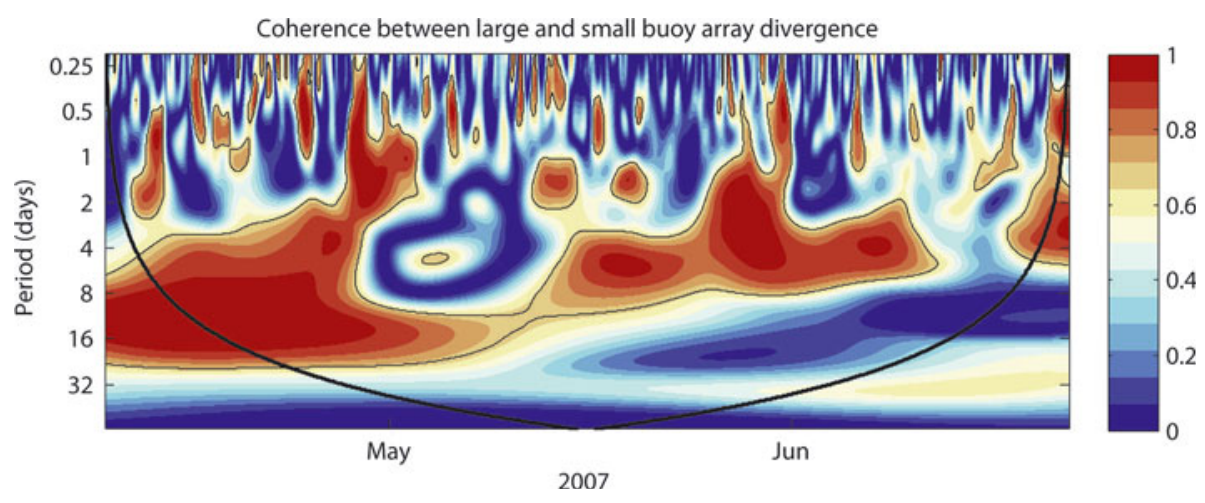

Fig. 11. The coherence between wavelet spectra of divergence time series for small $(20 \mathrm{~km})$, and large $(140 \mathrm{~km})$ buoy arrays. $95 \%$ significance levels are encircled by solid lines, and the cone of influence is shown with a bold solid line. 00:00Z on 1 May 1 and 1 June are indicated by their month.

responding in a coherent fashion to longer-range forcing than local synoptic events. During the 16 day period during which coherence is experienced, several different weather systems impacted ice dynamics in the Beaufort Sea. We can infer the length scale of influence for storms impacting ice in this region during late winter is much larger than the scale of the actual storm.

4. Around mid-May, coherence is lost over time periods longer than the synoptic period. This indicates a transition between winter and spring pack, with the connectivity of the pack reducing in spring. This transition occurs during one synoptic event, when the ice pack was observed to open. A high-pressure ridge system sat over the Beaufort Sea around 15 May, leading to a diverging ice pack, probably under clear skies. This was the first event, after the return of sunlight, that allowed substantial solar heating of the upper ocean through leads, and probably heralded the start of spring in the southern Beaufort Sea. It is interesting that after this single event, coherence was never regained over long time periods; connectivity of the ice pack had broken down during the high-pressure event, such that storms at a distance no longer impacted sea-ice deformation at the SEDNA site.

We find ice-pack divergence is coherent over synoptic timescales, over distances of $140 \mathrm{~km}$. In winter, fortnightly coherence timescales are observed, suggesting a coherence length scale of order $1000 \mathrm{~km}$. This large-scale coherence breaks down rapidly in spring during one synoptic event. Coherence is small over periods less than the synoptic timescale. We also see an increase in deformation variability and whitening of the deformation spectra at smaller scales less than $140 \mathrm{~km}$. This indicates that the deformation of a smaller-scale, $<140 \mathrm{~km}$ scale region embedded in a larger region cannot be predicted from deformation observations at the larger scale.

In winter, a connected ice pack will be consolidated and allow transmission of internal ice stress over large (regionalscale) distances, leading to coherent behaviour of the icepack strain-rate components over these distances. We estimate that this coherence distance is of the order $1000 \mathrm{~km}$ during late winter, but coherence breaks down below the synoptic scale. After the transition (around 15 May) the coherent length scale is similar to the synoptic length scale in the atmosphere (hundreds of kilometres). However, there were times when the small buoy-array divergence was not coherent to the large array divergence over synoptic periods. Our analysis indicates the notion of a decorrelation length scale for ice-pack divergence does not explain all spatial variability in divergence fields. Divergence is incoherent and hence uncorrelated between temporal scales smaller than the atmospheric synoptic scale and between spatial scales less than $100 \mathrm{~km}$, but displays coherence across larger scales.

\section{DISCUSSION AND CONCLUDING REMARKS}

In this paper, we produce findings in agreement with Marsan and others (2004) and Rampal and others (2008) using a set of ice-drifting buoys that were deployed in an array, designed for investigating temporal and spatial scaling in sea-ice deformation. The array design allows deformation scaling behaviour, and changes in coherence of deformation events between large and small spatial scales, to be related to weather. We find similar scaling relationships to Marsan and others (2004), Rampal and others (2008) and Stern and Lindsay (2009) with these data, and we identify the lengthand timescales over which the error characteristics of our deformation analysis allow representation of the scaling relationship. We highlight that the multi-fractal nature (demonstrated by Marsan and others, 2004) of deformation is important in characterizing its statistical properties. Time series of deformation is a pink-noise process, which indicates it is controlled by a balance between external forcing and internal dissipation. We suggest spectral and wavelet methods may be more appropriate for scaling analysis of sea-ice deformation, compared to the methods of Marsan and others (2004), Rampal and others (2008) and Stern and Lindsay (2009), and find that dissipative processes become more apparent as the region over which divergence is estimated decreases.

We investigated how position measurement resolution (sampling interval in space and time) can impact estimates of ice growth over winter, and find that, to correctly simulate ice growth, deformation must be resolved at the lead scale with at least hourly time sampling. However, the fact that deformation scales with spatial and temporal sampling rate, in a well-behaved way, suggests the statistical properties of deformation at small scales can be modelled given largerscale measurements. This would allow estimation of integral properties of the deformation field (e.g. end-of winter firstyear ice thickness) to be improved. 
It is intuitive that the scaling properties of deformation should be related to the coherence length scale experienced by the ice pack. Hutchings and others (unpublished information) found that the correlation in deformation time series between $10 \mathrm{~km}^{2}$ areas of ice reduces in variance as the distance between ice areas increases. The data were collected in early summer, and suggested that deformation correlation was lost at distances greater than the synoptic scale. In our experiment, we use cross-wavelet coherence analysis to investigate how coherence length scale is related to weather. An opening ice pack, with predominately anticyclonic forcing, displays lower coherence length scale than a closing ice pack, under predominately cyclonic forcing. Classic decorrelation length-scale analysis is an inappropriate metric for sea-ice deformation. The randomness, or degree of whiteness, of deformation time series increases as spatial scale decreases. At sub-synoptic scales, deformation does not display coherence across regions of the ice pack. Coherence is apparent over synoptic scales and over longer periods when the ice pack is predominately closing.

Rampal and others (2008) show scaling properties of deformation are different in summer and winter. Stern and Lindsay (2009) find an annual cycle in the fractal dimension of deformation, and find differences between perennial and seasonal ice packs. These two papers led us to postulate that these changes in fractal dimension could be related to changes in coherence length scale of deformation. However, when we tested this assumption by performing our scaling analysis over two separate periods (before and after 15 May) we did not find significant changes in fractal dimension between the two periods, though there is some indication the spectra may be whitened in the later period. This whitening follows the results of Rampal and others (2008) who found whitening in summer compared to winter, which indicates a loss of coherence in deformation, and a more homogeneous deformation field. Our results suggest that coherent behaviour of the ice pack, over synoptic to regional scales, is controlled by weather during the winter-to-spring transition. At sub-synoptic scales, where coherence is not an emergent property of the deformation field, deformation displays localization and fractal spatio-temporal scaling. The fact that deformation scales log-log linearly with spatial scale (Fig. 3) suggests that the mechanism of fracture for ice pack, from 100 to $20000 \mathrm{~km}^{2}$ in size, is consistent (Weiss, 2001) (following the theory of Bažant (1995) that demonstrates under quasi-brittle fracture the energy dissipated at cracks follows a fractal distribution). We find that Overland and others' (1995) observation that there is a hierarchy of scales for the arctic sea-ice deformation can be explained by the coherence in deformation over synoptic to regional scales, which results from wind stress being the primary driver in the sea-ice momentum balance.

Our results are consistent with an ice pack that deforms in response to changing synoptic forcing. Over the regional scale of the Beaufort Sea, fractures (leads and ridges) are organized into coherent patterns that are controlled by the confining stress of the pack which is related to wind stress and coastline geometry (see, e.g., Hutchings and others, 2005). During a particular storm, the ice pack fractures, and the active leads demonstrate a fractal distribution in space (Weiss and Marsan, 2004). These leads have a fractal distribution of deformation rates in time and space, and coherence in deformation across many leads only becomes apparent at synoptic scales. It is of note that the temporal scaling of deformation suggests non-equilibrium self-organized critically. The distribution of lead and ridge deformation magnitudes is an emergent property, related to the internal dynamics of the pack-ice momentum balance. Below the synoptic scale, sea-ice deformation is important in the ice mass balance, yet cannot be described as coherent with wind forcing and the larger-scale, $>100 \mathrm{~km}$, deformation field.

This has implications for the design of sea-ice deformation monitoring systems. If one is interested in integral properties, such as the magnitude of moisture and heat released from the ocean to atmosphere or the mass of first-year ice grown over winter, it should be possible to estimate these given an array of drifting buoys scattered across the Arctic to resolve synoptic scales. Process studies that need time series of these fluxes to be resolved require a different strategy. For these studies, one must monitor sea-ice deformation over the spatial scales that impact local measurements, resolving leads with at least hourly temporal resolution.

There are also implications for sea-ice modelling. Lowresolution models currently consider coherent deformation below the grid resolution through a viscous assumption (Hibler, 1977). This is clearly incorrect, and a new parameterization for sub-gridscale deformation is required if these models are to simulate ice growth and ocean-iceatmosphere fluxes sufficiently. Eddy-resolving Arctic iceocean models are being developed at high resolution $(\sim 2 \mathrm{~km})$, and these could simulate individual leads. Such models will have to pay close attention to the distribution of leads, which can be controlled through tuning of the icestrength parameterization (Hutchings and others, 2005). For coarser-resolution circulation models including sea ice it is important the sea-ice component represents lead to synoptic-scale deformation as described in this paper, and that coupling between ocean, ice and atmosphere is performed at least hourly.

It is difficult to estimate small-resolution strain rate at a particular location based on larger-scale measurements, because sea ice does not exhibit a classic decorrelation length scale and the statistical properties of ice-pack divergence are not stationary in time and space. Models that reproduced observed spatial scaling of divergence and leads, observed timescales of deformation variability, and the seasonally changing spatial scales over which deformation is coherent, may lead to improvements in representation of the sea-ice thickness distribution and oceanatmosphere fluxes. The dataset presented in this paper could be used to validate such a model (available at http:// dw.sfos.uaf.edu/sedna/).

\section{ACKNOWLEDGEMENTS}

Funding for this project has been provided by grant 0612527 from the US National Science Foundation (NSF) Office of Polar Programs. We express our deep gratitude to F. Karig and his team from the University of Washington Applied Physics Laboratory who directly supported our work in the field. This experiment would not have occurred without the support of J. Gosset, the Arctic Submarine Laboratory and the US Navy. We thank Pat McKeown, 'Andy' Anderson and Randy Ray who deployed our GPS ice drifters. The wavelet cross-coherence code base was provided by A. Grinsted and has been modified for the purpose of this study. Any opinions, findings and 
conclusions or recommendations expressed in this material are those of the authors and do not necessarily reflect the views of the NSF. The International Space Science Institute, Bern, Switzerland, is acknowledged for supporting this study, via project No. 169, through travel support for the authors to meet in person. An anonymous reviewer provided feedback that led to improvements to the paper, for which we are grateful. J. Hutchings thanks D. Newman for insightful conversations that helped in the development of the paper. C. Geiger also wishes to thank the Max Planck Institute for Solar System Research in Katlenburg-Lindau, Germany, for hospitality and support to complete the manuscript during the 2010 summer.

\section{REFERENCES}

Bažant, Z.P. 1995. Scaling of quasi-brittle fracture and the fractal question. J. Eng. Mater. Technol., 117(4), 361-367.

Geiger, C.A. and M.R. Drinkwater. 2001. Impact of temporalspatio resolution on sea-ice drift and deformation. In Dempsey, J.P. and H.H. Shen, eds. IUTAM Symposium on Scaling Laws in Ice Mechanics and Ice Dynamics, 13-16 June 2000, Fairbanks, Alaska. Proceedings. Dordrecht, Kluwer Academic, 407-416.

Geiger, C.A. and M.R. Drinkwater. 2005. Coincident buoy- and SAR-derived surface fluxes in the western Weddell Sea during Ice Station Weddell 1992. J. Geophys. Res., 110(C4), C04002. (10.1029/2003JC002112.)

Geiger, C.A., Y. Zhao, A.K. Liu and S. Häkkinen. 2000. Large-scale comparison between buoy and SSM/I drift and deformation in the Eurasian Basin during winter 1992-1993. J. Geophys. Res., 105(C2), 3357-3368.

Grinsted, A., J.C. Moore and S. Jevrejeva. 2004. Application of the cross wavelet transform and wavelet coherence in geophysical time series. Nonlinear Proc. Geophys., 11(5-6), 561-566.

Hibler, W.D., III. 1977. A viscous sea ice law as a stochastic average of plasticity. J. Geophys. Res., 82(27), 3932-3938.

Hutchings, J.K., ed. 2009. The Sea Ice Experiment: Dynamic Nature of the Arctic (SEDNA). Fairbanks, AK, University of Alaska. International Arctic Research Centre. Applied Physics Laboratory Ice Station. (APLIS 2007 Field Report IARCTP09-0001.)
Hutchings, J.K., P. Heil and W.D. Hibler, III. 2005. Modeling linear kinematic features in sea ice. Mon. Weather Rev., 133(12), 3481-3497.

Hutchings, J.K. and 15 others. 2008. Exploring the role of ice dynamics in the sea ice mass balance. Eos, 89(50), 515-516.

Jenkins, G.M. and D.G. Watts. 1969. Spectral analysis and its applications. London, Holden-Day.

Kwok, R. 2001. Arctic Ocean sea ice deformation from SAR ice motion: linear kinematic features. Pasadena, CA, NASA Jet Propulsion Laboratory. (Tech. Rep. JPL D-21524.)

Lindsay, R.W. and H.L. Stern. 2003. The RADARSAT geophysical processor system: quality of sea ice trajectory and deformation estimates. J. Atmos. Oceanic Technol., 20(9), 1333-1347.

Marsan, D., H. Stern, R. Lindsay and J. Weiss. 2004. Scale dependence and localization of the deformation of Arctic sea ice. Phys. Rev. Lett., 93(17), 178501. (10.1103/PhysRevLett.93.178501.)

Maykut, G.A. and N. Untersteiner. 1971. Some results from a timedependent thermodynamic model of sea ice. J. Geophys. Res., 76(6), 1550-1575.

McNutt, S.L. and J.E. Overland. 2003. Spatial hierarchy in Arctic sea ice dynamics. Tellus A, 55(2), 181-191.

Overland, J.E., B.A. Walter, T.B. Curtin and P. Turet. 1995. Hierarchy and sea-ice mechanics: a case study from the Beaufort Sea. J. Geophys. Res., 100(C3), 4559-4571.

Rampal, P., J. Weiss, D. Marsan, R. Lindsay and H. Stern. 2008. Scaling properties of sea ice deformation from buoy dispersion analysis. J. Geophys. Res., 113(C3), C03002. (10.1029/ 2007JC004143.)

Schulson, E.M. and W.D. Hibler, III. 1991. The fracture of ice on scales large and small: Arctic leads and wing cracks. J. Glaciol., 37(127), 319-322.

Stern, H.L. and R.W. Lindsay. 2009. Spatial scaling of Arctic sea ice deformation. J. Geophys. Res., 114(C10), C10017. (10.1029/ 2009JC005380.)

Walter, B.A. and J.E. Overland. 1993. The response of lead patterns in the Beaufort Sea to storm-scale wind forcing. Ann. Glaciol., 17, 219-226.

Weiss, J. 2001. Fracture and fragmentation of ice: a fractal analysis of scale invariance. Eng. Fract. Mech., 68(17-18), 1975-2012.

Weiss, J. and D. Marsan. 2004. Scale properties of sea ice deformation and fracturing. C. R. Phys., 5(7), 735-751. 